\title{
Improving estimates for reliability and cost in microgrid investment planning models
}

Cite as: J. Renewable Sustainable Energy 11, 045302 (2019); https://doi.org/10.1063/1.5094426

Submitted: 01 March 2019 . Accepted: 25 June 2019. Published Online: 16 July 2019

Ryan Hanna, Vahid R. Disfani, Hamed Valizadeh Haghi (D), David G. Victor, and Jan Kleissl (D)
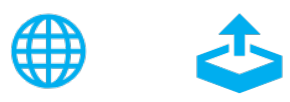

View Online

\section{ARTICLES YOU MAY BE INTERESTED IN}

The potential role of green hydrogen production in the South Africa energy mix

Journal of Renewable and Sustainable Energy 11, 044301 (2019); https://

doi.org/10.1063/1.5089958

Passive solar tracker based in the differential thermal expansion of vertical strips

Journal of Renewable and Sustainable Energy 11, 043701 (2019); https://

doi.org/10.1063/1.5100492

Air gap membrane distillation: A review

Journal of Renewable and Sustainable Energy 11, 045901 (2019); https://

doi.org/10.1063/1.5063766

\section{AIP Author Services English Language Editing}




\title{
Improving estimates for reliability and cost in microgrid investment planning models
}

\author{
Cite as: J. Renewable Sustainable Energy 11, 045302 (2019); doi: 10.1063/1.5094426 \\ Submitted: 1 March 2019 • Accepted: 25 June 2019 . \\ Published Online: 16 July 2019

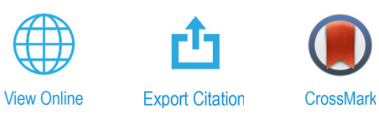

\author{
Ryan Hanna, ${ }^{1,2, a)}$ Vahid R. Disfani, ${ }^{3}$ Hamed Valizadeh Haghi, ${ }^{4}$ (D) David G. Victor, and Jan Kleissl ${ }^{4,5}$ (D)

\section{AFFILIATIONS} \\ ${ }^{7}$ School of Clobal Policy and Strategy, University of California San Diego, La Jolla, California 92093, USA \\ ${ }^{2}$ International Institute for Applied Systems Analysis (IIASA), Laxenburg, Austria \\ ${ }^{3}$ Department of Electrical Engineering, University of Tennessee at Chattanooga, Chattanooga, Tennessee 37403, USA \\ ${ }^{4}$ Center for Energy Research, University of California San Diego, La Jolla, California 92093, USA \\ ${ }^{5}$ Department of Mechanical and Aerospace Engineering, University of California San Diego, La Jolla, California 92093, USA \\ a)rehanna@ucsd.edu
}

\begin{abstract}
This paper develops a new microgrid investment planning model that determines cost-optimal investment and operation of distributed energy resources (DERs) in a microgrid. We formulate the problem in a bilevel framework, using particle swarm optimization to determine investment and the DER-CAM model (Distributed Energy Resources Customer Adoption Model) to determine operation. The model further uses sequential Monte Carlo simulation to explicitly simulate power outages and integrates time-varying customer damage functions to calculate interruption costs from outages. The model treats nonlinearities in reliability evaluation directly, where existing linear models make critical simplifying assumptions. It combines investment, operating, and interruption costs together in a single objective function, thereby treating reliability endogenously and finding the cost-optimal trade-off between cost and reliability-two competing objectives. In benchmarking against a version of the DER-CAM model that treats reliability through a constraint on minimum investment, our new model improves estimates of reliability (the loss of load expectation) by up to $600 \%$, of the total system cost by $6 \%-18 \%$, of the investment cost by $32 \%-50 \%$, and of the economic benefit of investing $27 \%-47 \%$. Improvements stem from large differences in investment of up to $56 \%$ for natural gas generators, solar photovoltaics, and battery energy storage.
\end{abstract}

Published under license by AIP Publishing. https://doi.org/10.1063/1.5094426

\section{INTRODUCTION}

Microgrids are widely touted for the benefits they can confer to both public and private stakeholders. They can improve reliability and resiliency, lower energy costs, and reduce the carbon intensity of energy, among others. ${ }^{1-4}$ Numerous state governments have enacted microgrid grant programs, targeting reliability and resiliency, in particular, e.g., in Connecticut, ${ }^{5}$ Massachusetts, ${ }^{6,7}$ New Jersey, ${ }^{8}$ California, ${ }^{9,10}$ New York, ${ }^{11}$ and Maryland. ${ }^{12}$ Other states still, such as Minnesota ${ }^{13}$ and Rhode Island, ${ }^{14}$ have convened task forces to explore opportunities for microgrids. Deployment rates are increasing in the private sector as well, ${ }^{15-17}$ driven by customer concerns about reliability, falling technology costs, and opportunities to reduce electric utility bills.

Yet, microgrids remain costly. Identifying benefits-and concomitant revenue streams-is therefore critical. To ensure positive return on investment, multiple (potentially all) available benefits may need to be considered, e.g., by "value stacking." ${ }^{18,19}$ Identifying benefits early in the design process is important because investment and benefits are inextricably linked: the choice of distributed energy resources (DERs) affects the magnitude of benefits that can be realized, and, likewise, value streams known a priori (and considered) affect the optimal selection of DERs. One challenge, however, is that certain benefits are hard to quantify-notably reliability. There is little agreement on how reliability should be valued, and many are grappling with the conceptual challenge. $^{20-22}$ Reliability poses modeling challenges as well due to its nonlinear form; as such, models vary widely in their treatment of reliability, and simplifications are common.

In this paper, we address microgrid "investment planning" models-economic-engineering optimization models that assess technical and economic feasibility and whose core function is to select DERs that optimize an objective, such as minimizing the total system cost, power outages, or emissions. ${ }^{23}$ While these models have the capacity to address the many facets of reliability ${ }^{24}$ simultaneouslye.g., bulk grid outages, individual points of hardware failure, scheduled 
downtime, reliability costs and constraints, and a customer's valuation of reliability-few have been built to do so.

Numerous investment planning models have been developed, including several sponsored by the U.S. Department of Energy (DOE): HOMER (Hybrid Optimization of Multiple Energy Resources), ${ }^{25}$ DERCAM (Distributed Energy Resources Customer Adoption Model), ${ }^{26}$ MDT (Microgrid Design Toolkit), ${ }^{27}$ and REopt. ${ }^{28}$ The four DOE models are functionally similar: they use cost-benefit analysis to assess technical and economic performance, determine least-cost investment and operation of DERs, and estimate energy provision, fuel consumption, emissions, and costs (component costs, levelized cost of energy, and net present cost). ${ }^{29}$ The models are structurally similar as well. They are based on optimization and thus consist of three main parts: an objective function that enumerates system costs or other performance criteria such as emissions or reliability; decision variables for DER investment and operation that minimize the objective function; and constraints on decision variables that respect technological and economic limitations. Though the models differ in small ways in each of these three domains, their principal difference lies in their formulation of the objective function and, in particular, their treatment of reliability therein.

HOMER lacks a reliability cost, which represents a customer's economic losses from power outages, in its objective function. Instead, it generates grid outage sequences-i.e., down-times when the bulk grid is failed-which force a microgrid to operate autonomously in the islanded mode. Without reliability costs, there is no cost associated with experiencing outages nor a benefit (avoided economic losses) from preventing them. Outages thus act only as constraints on operation.

DER-CAM, by contrast, includes a reliability cost in its objective function. ${ }^{30}$ The reliability cost is formulated as the product of unserved (or curtailed) load due to grid outages and the value of lost load (VOLL), a dollar per kilowatt-hour measure, where the timing and duration of outages are prescribed. Given the schedule of outages, the model finds whether it is more economic to forego DER investment and incur cost due to unserved load or to invest in additional DERs to mitigate the cost of unserved load during outages. DER-CAM further addresses reliability via a constraint on investment that requires the aggregate DER capacity, measured in kilowatts, exceed the peak critical load.

MDT also includes a reliability cost in its objective function, combining reliability metrics, such as outage frequency and unserved load, with other metrics like system cost, fuel use, and system efficiency using multiobjective optimization. However, MDT lacks an integrated framework for treating reliability because it couples two disparate models. The first, called the Microgrid Sizing Capability (MSC) module (which is a tuned version of DER-CAM), determines optimal DER investment without a reliability objective. The second model, the Technology Management Optimization (TMO) module, then refines DER-CAM's result by including other objectives, such as reliability, efficiency, and grid hardware costs. Because reliability is not included in the initial optimization routine, it is largely exogenous to the model. A more robust approach would be to include reliability costs in a single objective function with all other system costs.

REopt includes a reliability cost in the objective function and, in attempting to handle its nonlinearities, uses linear regression to relate DER capacity to reliability, specifically the probability that a microgrid can maintain power in the islanded mode during a prescribed grid outage. The unmet load due to outages incurs a cost per a prescribed
VOLL. At present, the framework is only fit for use with solar-plusstorage configurations and a single average outage duration.

The disparate and patchwork treatment of reliability in the DOE models is due, fundamentally, to the fact that reliability is highly nonlinear, and yet the DOE models are built on mixed integer "linear" programming (MILP). The objective of this work is to rectify this incompatibility using appropriate methods. Our approach is to formulate a new model that is functionality identical to the DOE models but that is structured, first, on best-available methods for calculating reliability. That is, we set out to use the most accurate reliability evaluation method available-sequential Monte Carlo simulation (MCS) ${ }^{31}$ - and, only afterward, to incorporate existing MILP elements where sensible. That reorientation is simple conceptually but demands an entirely new model.

We propose a bilevel framework consisting of heuristic optimization, specifically particle swarm optimization (PSO) in the upper level and DER-CAM in the lower level. The PSO sizes DERs and DERCAM schedules them. Also in the lower level is the MCS routine ${ }^{32}$ that calculates reliability. PSO is highly flexible and can incorporate nonlinearities in objective and constraint functions, thereby overcoming the central modeling challenge of reliability. MCS, also highly flexible, can incorporate random grid outages, prescribed outages, scheduled DER maintenance, random DER failures, and widespread (dependent) outages to the grid and particular DERs. Our model further makes use of duration- and time-dependent customer damage functions ${ }^{33}$ to value reliability, in contrast to existing models that use VOLL. ${ }^{34-39}$ VOLL is a simplified representation of a customer's value of reliability but is commonly used because it fits with the mathematical framework of MILP.

Our approach is most similar to the MDT model, which also uses heuristic optimization, namely a genetic algorithm, as well as simulation to calculate reliability. However, our approach treats reliability and investment decision-making together in a single integrated framework, in contrast to MDT's use of two disparate models. We thus treat reliability endogenously with all other system costs. To our knowledge, our model is the first that addresses the investment planning problem using an integrated framework consisting of optimization and sequential MCS.

As we discuss (Sec. II), investment planning models can (and should) be structured to explicitly include many real-world phenomena that affect customer reliability and that therefore drive investment choices-such as bulk grid outages, random DER failures, maintenance, and the cost and value of reliability. Our new framework (Sec. III) includes each. We validate our new model against DER-CAM and, using case studies (Sec. IV), show how our new model improves estimates of investment, reliability, cost, and benefit compared to DERCAM's use of a reliability constraint. The comparisons illustrate how a cost-based approach to integrating reliability drives differences in key results compared to a constraint-based approach. We conclude (Sec. $\mathrm{V})$ by looking forward to logical extensions of the work.

\section{PROBLEM FORMULATION}

The central objective of the microgrid investment planning problem is to select, size, and schedule DERs to minimize the total system cost, which includes the expected cost of investment, operation, and interruption. ("Interruption cost" and "reliability cost" are used here interchangeably, though in the field of reliability evaluation the former is preferred, while the latter is better described as an "unreliability" 
cost.) An electric utility customer's operating cost can be reduced by self-generating less costly energy on-site, while the interruption cost (i.e., a customer's economic losses from power outages) can be mitigated by investing in a reliable DER mix that can island and sustain power independent of the bulk grid. The solution to the problem is an optimally sized set of DERs and their operating schedules, an enumeration of all costs (and subsequent benefits), and an optimal level of reliability. We refer to this optimal DER mix as the "microgrid configuration."

\section{A. Microgrid customer topology}

We consider the utility customers who may pursue microgrids to be grid-connected at the distribution level, with all load and DERs located at a single bus on the load side of the distribution transformer [Fig. 1(a)]. From the modeling standpoint, customers may comprise one or several buildings; from the regulatory standpoint, they are single utility customers with a single electric billing meter at the point of common coupling (PCC). Our focus is single customers because rules governing U.S. electric utilities in most jurisdictions prohibit microgrids from serving multiple adjacent customers whose properties span public spaces, such as roads.

Customer loads can be supplied by a combination of grid electricity and self-generated power. Inside the microgrid, sources of energy provision fall broadly into four categories:

1. Natural gas-fired generators: internal combustion engines and microturbines with fixed capacity that can include heat recovery;

2. Renewables: solar photovoltaics (PV);

3. Electric storage: stationary battery energy storage; and

4. Thermal resources: natural gas-fired chillers, absorption chillers that affix generators with heat recovery, and thermal energy storage (hot and cold).
Technologies are modeled per their technical specifications (capacity, dispatch, ramping, and efficiency ratings) as well as those for cost (fixed costs, variable costs, and lifetime) and reliability (failure rate and repair rate). The bulk grid is modeled as a single equivalent generator [Fig. 1(b)]-appropriate treatment because the microgrid does not impact grid resource adequacy and because the bulk grid acts as a homogeneous system capable of supplying peak microgrid loads. ${ }^{40}$ Grid electricity costs are defined in the utility tariff, while grid failure and repair rates are a function of the utility metrics SAIFI (system average interruption frequency index), MAIFI (momentary average interruption frequency index), and SAIDI (system average interruption duration index).

We model the combined system at a single node, thereby framing a hierarchical level I reliability evaluation, also referred to as resource adequacy. ${ }^{24,41}$ Recent modeling (e.g., with MDT and DER-CAM) has begun to add elements of power system networks, such as power system equipment, constraints, and power flow equations. Though multinodal analysis is outside the scope of this work because our principal focus is resource adequacy, network capacity constraints could be modeled, e.g., by including appropriate power flow equations, as well as bus voltage and ampacity limits for lines and power equipment, as constraints in the problem, thereby capping power flow from individual DERs or the bulk grid.

\section{B. Investment and operating costs}

Investment and operating costs are standard components of investment planning models. The investment cost is the annualized, amortized capital cost of investing in DERs and the switching equipment that enables islanding,

$$
c_{\text {investment }}:=c_{\text {switch }}+c_{\text {gen }}+c_{\mathrm{pv}}+c_{\mathrm{es}}+c_{\text {thermal }} \text {, }
$$

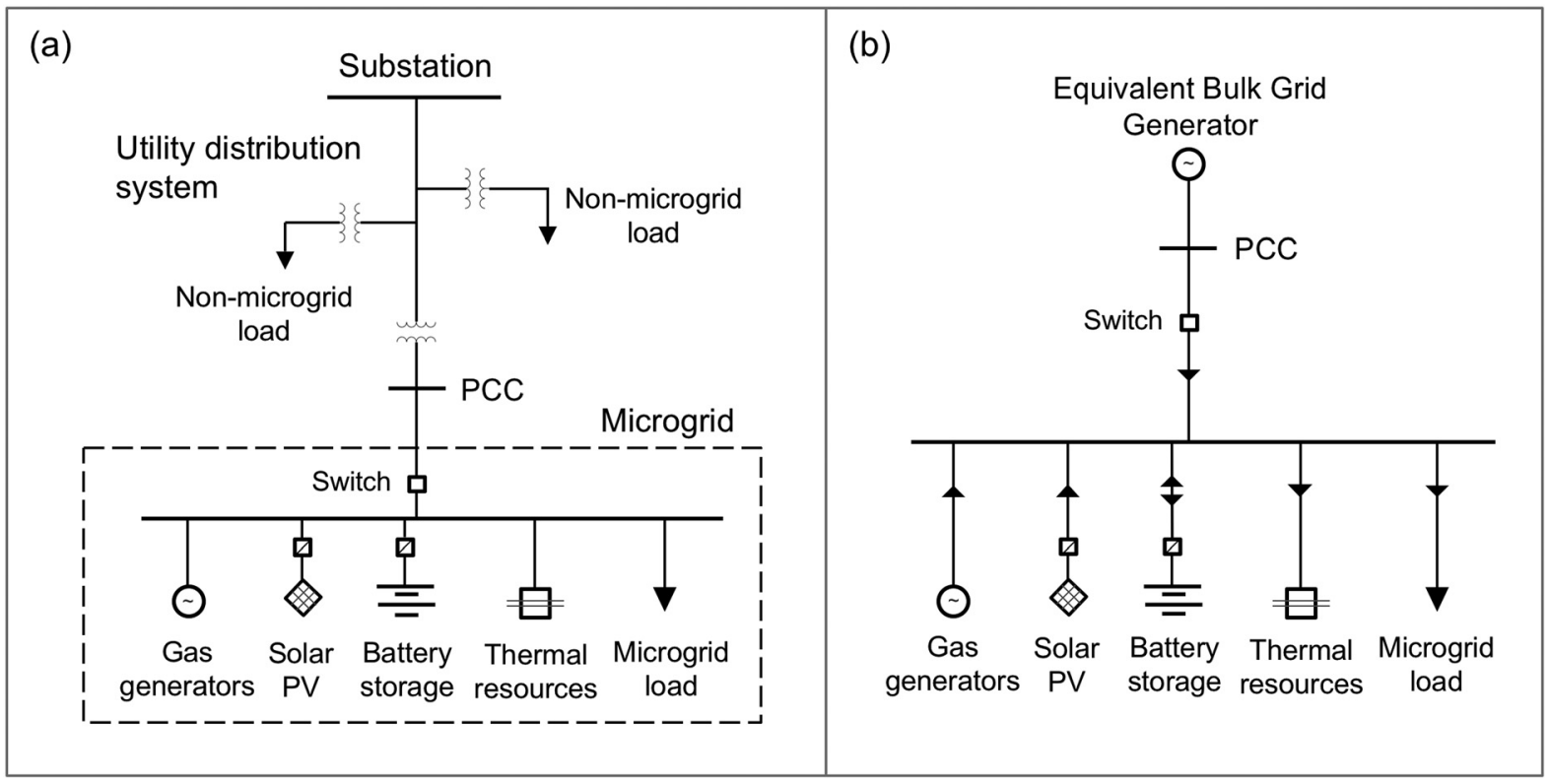

FIG. 1. (a) The distribution system-microgrid topology and (b) the modeled microgrid system. In the modeled system, the distribution system is a single "equivalent" generator. DERs lie behind the point of common coupling (PCC) and comprise the four categories shown. (Thermal networks and the natural gas network are not shown.). 
where "switch" denotes switching equipment, "gen" generators, "pv" solar PV, "es" battery energy storage, and "thermal" thermal resources. $c_{\mathrm{gen}}$ is the sum of individual discrete generator costs; $c_{\mathrm{switch}}, c_{\mathrm{pv}}$, and $c_{\mathrm{es}}$ are the costs of single systems sized in any continuous capacity; and $c_{\text {thermal }}$ is the sum of costs for gas-fired chillers, absorption chillers, heat storage, and cold storage,

$$
\begin{aligned}
& c_{\text {switch }}:=A_{s} \operatorname{Bin}_{s}\left(C f c a p_{s}+C v c a p_{s} P_{\text {PurchCap }}\right), \\
& c_{\text {gen }}:=A_{i} \sum_{i \in I} \text { PurchNum }_{i} R_{i} \text { Cvcap }_{i},
\end{aligned}
$$

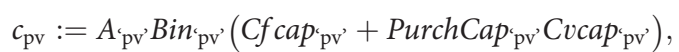

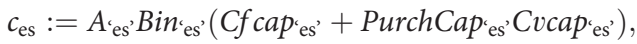

$$
\begin{aligned}
& c_{\text {thermal }}:=\sum_{k \in K} \text { PurchNum }_{k} R_{k} \operatorname{Cvcap}_{k} A_{k} \\
& +\sum_{q \in Q}\left(\operatorname{Bin}_{q} C f c a p_{q}+\operatorname{PurchCap}_{q} \operatorname{Cvcap}_{q}\right) A_{q},
\end{aligned}
$$

where $q$ here indexes absorption chillers "ac," heat storage "hs," and cold storage "cs." Nomenclature for the investment and operating cost calculations is given in Table I. The annuity factor $A$ for technology $v$ is given by

$$
A_{v}=\frac{\text { IntRate }}{1-\frac{1}{(1+\text { IntRate })^{\text {Lifetime }_{v}}}},
$$

and IntRate is the interest rate.

The operating cost comprises costs incurred from system operation-from electricity purchases $c_{\text {tariff }}$, natural gas purchases $c_{\text {fuel }}$, resource maintenance $c_{\mathrm{der}}$, and emitting carbon $c_{\text {carbon, }}$,

$$
c_{\text {operating }}:=c_{\text {tariff }}+c_{\text {fuel }}+c_{\text {der }}+c_{\text {carbon }},
$$

where

$$
\begin{aligned}
& c_{\text {tariff }}:=\sum_{m \in M} \sum_{p \in P} \sum_{t \in T} \sum_{h \in H} \pi^{`} \mathrm{ds}^{\prime}, m, t, h, \cdot N_{m, t} \cdot V C h g_{m, p} \\
& +\sum_{m \in M} \sum_{d \in D} D C h g_{m, d} \cdot \max _{t \in T, h \in d}\left\{\pi^{\prime} d s^{\prime}, m, t, h\right\}+\sum_{m \in M} \text { ElecFee } \\
& +\sum_{m \in M}\left(\sum_{i \in I} \text { Purch Num }_{i} \cdot R_{i}+\text { PurchCap'pv' }^{\prime}\right) S C h g, \\
& c_{\text {fuel }}:=\sum_{m \in M} \text { NGFee }+\sum_{m \in M} \sum_{t \in T} \sum_{h \in H} \gamma_{m, t, h} \cdot N_{m, t} \cdot \text { NGPrice }_{m} \text {, } \\
& c_{\mathrm{der}}:=\sum_{i \in I} \sum_{m \in M} \text { PurchNum }_{i} \cdot R_{i} \cdot \frac{\text { Cfom }_{i}}{12} \\
& +\sum_{i \in I} \sum_{m \in M} \sum_{t \in T} \sum_{h \in H} \pi_{i, m, t, h} \cdot N_{m, t} \cdot \text { Cvom }_{i} \\
& +\sum_{k \in K} \sum_{m \in M} \text { PurchNum }_{k} \cdot R_{k} \cdot \frac{\text { Cfom }_{k}}{12} \\
& +\sum_{k \in K} \sum_{m \in M} \sum_{t \in T} \sum_{h \in H} \xi_{k, m, t, h} \cdot N_{m, t} \cdot \text { Cvom }_{k} \\
& +\sum_{q \in Q} \sum_{m \in M} \text { PurchCap }_{q} \cdot \text { Cfom }_{q}
\end{aligned}
$$

\begin{tabular}{|c|c|}
\hline Parameter & Description \\
\hline \multicolumn{2}{|c|}{ Sets and indices } \\
\hline$M$ & Month, $M=\{1,2, \ldots, 12\}$ \\
\hline$T$ & Day-type, $T=\{$ week, weekend $\}$ \\
\hline$H$ & Hour, $H=\{1,2, \ldots, 24\}$ \\
\hline$P$ & Tariff period $P=\{$ on-peak, midpeak, off-peak $\}$ \\
\hline$D$ & $\begin{array}{l}\text { Tariff demand type, } D=\text { \{noncoincident, on-peak, } \\
\text { midpeak, off-peak }\}\end{array}$ \\
\hline$U$ & $\begin{array}{l}\text { End-use load, } U=\{\text { electricity "el," cooling "cl," space } \\
\text { heating "sh," water heating "wh," natural gas "ng" }\end{array}$ \\
\hline$s$ & Index for switchgear \\
\hline$i$ & Generator, $I=\{\mathrm{ICE}, \mathrm{MT}, \mathrm{ICE}-\mathrm{HX}, \mathrm{MT}-\mathrm{HX}\}^{\mathrm{a}}$ \\
\hline$k$ & Direct-fired chiller, $K=\{$ DFChiller-HX $\}$ \\
\hline$q$ & $\begin{array}{l}\text { Continuous DER, } Q=\{\text { solar PV "pv," electric storage } \\
\text { "es," absorption chiller "ac," heat storage "hs," cold } \\
\text { storage "cs" }\}^{\mathrm{b}}\end{array}$ \\
\hline$v$ & All microgrid technologies, $V=\{\mathrm{I}, \mathrm{K}, \mathrm{Q}$, switchgear $\}$ \\
\hline e & $\begin{array}{l}\text { Source of electricity, } E=\{\mathrm{I} \text {, "pv," "es," distribution } \\
\text { system "ds" }\}\end{array}$ \\
\hline$c$ & $\begin{array}{l}\text { Source of cooling, } C=\{K \text {, absorption chiller "ac," } \\
\text { electric chiller "ec," cold storage "cs" }\}\end{array}$ \\
\hline$g$ & Source of heat, $G=\{I$, direct fuel "di" $\}$ \\
\hline
\end{tabular}

TABLE I. Nomenclature for investment and operating cost calculations in Eqs. (1)-(12).

Customer load

$N_{m, t} \quad$ Number of days of day-type $t$ in month $m$

$L_{u, m, t, h} \quad$ Load profile for end-use load $u$, month $m$, day-type $t$, and hour $h, \mathrm{~kW}$

Tariff parameters

ElecFee Fee for electric service, \$/month

$V C h g_{m, p} \quad$ Volumetric charge for month $m$ and tariff period $p$,

$\$ / \mathrm{kWh}$

$D C h g_{m, d} \quad$ Demand charge for month $m$ and demand type $d$, $\$ / \mathrm{kW}$

SChg DER standby charge, $\$ / \mathrm{kW} /$ month

NGFee Fee for natural gas service, $\$ /$ month

NGPrice $_{m} \quad$ Natural gas price in month $m, \$ / \mathrm{kWh}$

DER parameters

$R_{v} \quad$ Nameplate capacity of technology $v, \mathrm{~kW}$

$C f c a p_{v} \quad$ Fixed capital cost of technology $v, \$$

Cvcap $\quad$ Variable capital cost for technology $v, \$ / \mathrm{kW}$ or $\$ / \mathrm{kWh}$

$C_{\text {fom }} \quad$ Fixed O\&M cost for technology $v, \$ / \mathrm{kW} / \mathrm{yr}$ for $I, K$

and $\$ / \mathrm{kW} /$ month, or $\$ / \mathrm{kWh} /$ month for $Q$

Cvom $_{v} \quad$ Variable O\&M cost for technology $v, \$ / \mathrm{kWh}$

Lifetime $_{v} \quad$ Operational lifetime of technology $v$

$\mathrm{CO}_{2}$ parameters

EF Natural gas $\mathrm{CO}_{2}$ emission factor, $\mathrm{tCO}_{2} / \mathrm{kWh}$

CTax Tax on $\mathrm{CO}_{2}$ emissions, $\$ / \mathrm{tCO}_{2}$

Selection and sizing decision variables

PurchNum $_{i}$ Number of purchased gas generators $i$ 
TABLE I. (Continued.)

\begin{tabular}{ll}
\hline \hline Parameter & \multicolumn{1}{c}{ Description } \\
\hline PurchNum $_{k}$ & Number of purchased direct-fired chillers $k$ \\
Bin $_{q}$ & Binary decision variable to invest in DER $q$ \\
Bin $_{s}$ & Binary decision variable to invest in switchgear \\
PurchCap $q_{q}$ & Capacity of installed DER $q, \mathrm{~kW}$ \\
PurchCaps $_{s}$ & Capacity of installed switchgear, $\mathrm{kW}$ \\
Scheduling variables, $\mathrm{d}$ & \\
$\pi_{e, m, t, h}$ & Electricity provision from source $e, \mathrm{~kW}$ \\
$\xi_{c, m, t, h}$ & Cooling provision from source $c, \mathrm{~kW}$ \\
$\gamma_{m, t, h}$ & Total natural gas purchased, $\mathrm{kW}$ \\
$\gamma_{i, m, t, h}$ & Natural gas purchased for gas generator $i, \mathrm{~kW}$ \\
$\gamma_{k, m, t, h}$ & Natural gas purchased for direct-fired chiller $k, \mathrm{~kW}$ \\
\hline \hline
\end{tabular}

${ }^{a}$ Notation: ICE-internal combustion engine, MT-microturbine, and -HX-with heat recovery.

${ }^{\mathrm{b}}$ The set $Q$ does not include the electric chiller (which consumes electricity to supply the cooling load) because it is installed in every model run and hence does not create differences between runs.

"Subscript " $m, t, h$ " denotes "month, day-type, hour."

${ }^{\mathrm{d}}$ Natural gas purchases are secondary variables (i.e., they are a function of scheduling decision variables).

$$
c_{\text {carbon }}:=\sum_{m \in M} \sum_{t \in T} \sum_{h \in H}\left(\sum_{i \in I} \gamma_{i, m, t, h}+\sum_{k \in K} \gamma_{k, m, t, h}\right) \cdot N_{m, t} \cdot \text { EF } \cdot \text { CTax. }
$$

\section{Interruption cost}

The interruption cost is the customer's total economic loss from outages, in dollars per year, and is given by

$$
c_{\text {interruption }}:=\mathbf{E}\left[\sum_{i} \bar{l}_{i} c_{k}\left(d_{i}\right)\right],
$$

where $\mathbf{E}[\cdot]$ denotes the expected value, $\bar{l}$ is the customer's average load, $c_{k}\left(d_{i}\right)$ is the interruption cost function (i.e., customer damage function) for customer type $k$, and $\mathrm{d}$ is the interruption duration. An expected value is used because the interruption cost is the mean of a distribution of interruption costs output by the MCS (as we will explain in Sec. III B 3).

Microgrids generate value by islanding during outages, thereby avoiding the economic losses that passive customers otherwise incur. The difference between the pre- and post-investment interruption cost is the economic value attributable to improved reliability.

\section{MODEL IMPLEMENTATION}

\section{A. Objectives and constraints}

We formulate the two planning tasks_-sizing and schedulinghierarchically in a bilevel optimization framework. This approach separates the problem into a sizing component and scheduling component, each with a distinct objective function and a set of decision variables. The upper level is the sizing problem and seeks to minimize the total system cost,

$$
\min \quad c_{\text {total }}:=c_{\text {investment }}+c_{\text {operating }}+c_{\text {interruption }} .
$$

Decision variables are sizing decisions for discrete gas generators, solar PV, and battery energy storage-the three types of DERs in the model that contribute to reliability. Upper-level constraints can include minimum and maximum bounds on DER capacities, minimum requirements for reliability and resiliency, and caps on investment capital and operating expenditure.

The lower level is the scheduling problem and minimizes the operating cost for the full configuration,

$$
\min \quad c_{\text {operating}} \text {, }
$$

which, after evaluation, is returned to the upper level in Eq. (14). Decision variables are the operational set-points for DERs and for purchases of electricity and natural gas. Lower-level constraints enforce supply-demand energy balances, as well as other physical constraints standard in operation, such as DER dispatch and ramping limits, energy conversion, and energy efficiencies. As we will explain in Sec. III B, we use DER-CAM to calculate the investment and operating cost, consistent with our previous work. ${ }^{42}$

\section{B. Bilevel model framework}

The bilevel model consists of four modules, as shown in Fig. 2: (1) the PSO sizes the DERs that generate power and hence contribute to reliability—gas generators, solar PV, and battery storage; (2) DERCAM sizes thermal resources and solves the scheduling problem; (3) the MCS routine simulates system behavior, including outages, to evaluate reliability for customers within the microgrid; and (4) the Lawrence Berkeley National Laboratory (LBNL) regression model defines the customer damage functions that monetize, or place a dollar value on, individual outages. The PSO module serves to integrate the other three in a single optimization framework. We detail each in the following subsections Sec. III B 1-III B 4.

The full set of decision variables includes DER sizing and scheduling, purchases of grid electricity, and purchases of natural gas. Energy demand is defined by distinct load profiles for electricity, heating, cooling, and natural gas. The model horizon is the first year of deployment, and the model time step is $1 \mathrm{~h}$. Key outputs include the full set of decision variables, as well as the optimal level of reliability (both the expected value and the full probability distribution) and hourly totals for costs, energy supply-demand, and emissions.

We have built our MCS routine to transfer data with DER-CAM. The hourly operating schedules determined by DER-CAM, which include generator operating states, PV power output, and battery stateof-charge, are passed to the MCS and used in sequential simulations as initial conditions when outages occur. At present, it is not possible to transfer data in the opposite direction, from the MCS to DER-CAM, and so DER-CAM estimates the operating cost without the MCS's set of outages. Though this produces some error in the reported operating cost, that error is a small fraction of the total operating cost because typical SAIDI values (hours of outage per year) for U.S. distribution systems are small ( $<10 \mathrm{~h}$, or $0.1 \%$ of the year). This error could be corrected by combining scheduling and reliability evaluation in a single submodel, but we leave that for future work. 


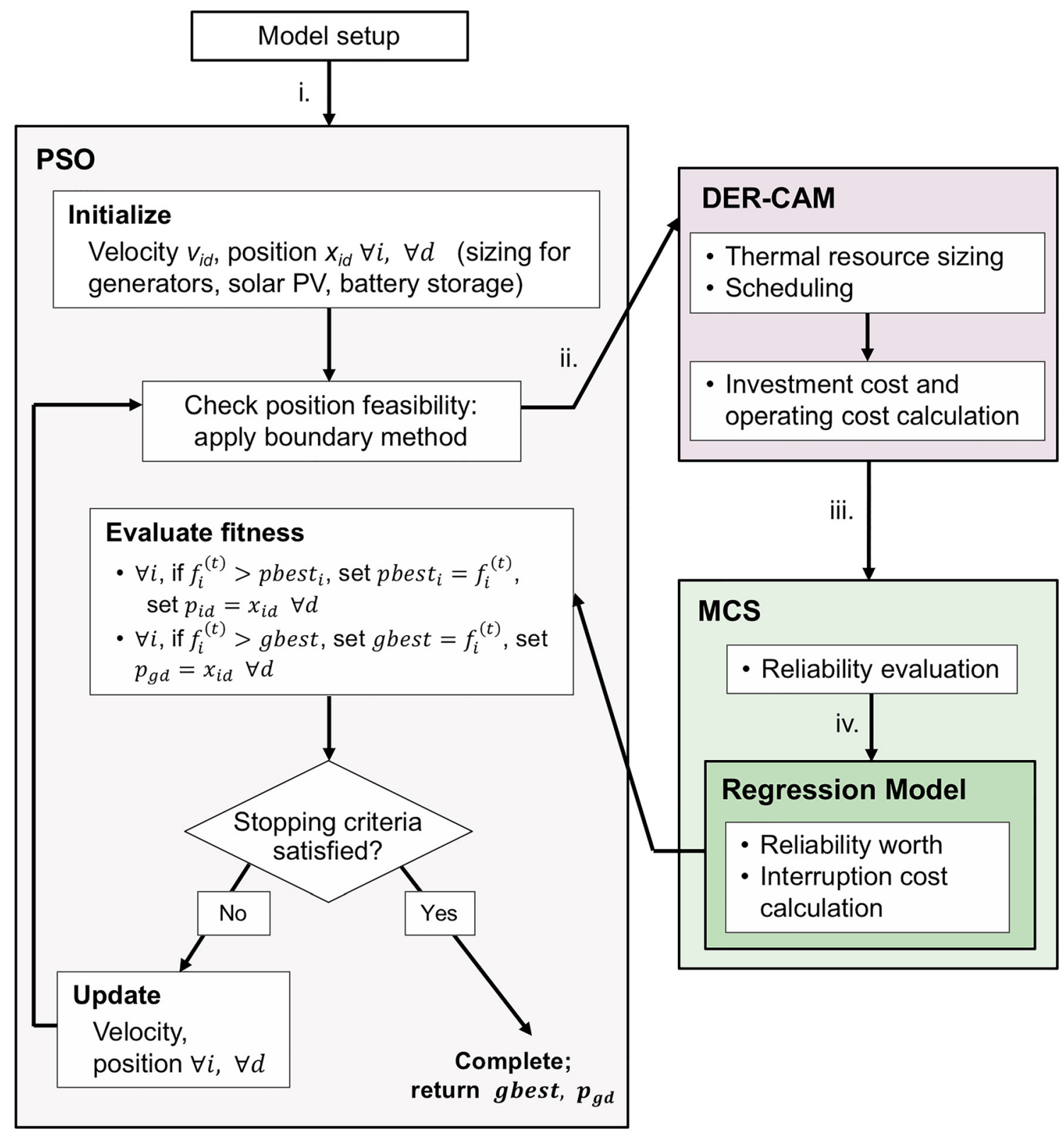

FIG. 2. The bilevel model consists of four modules: a PSO algorithm serves as the upper level, while DER-CAM, an MCS routine, and a regression model comprise the lower level. Four intermodule data transfers are notable: (i) first, the PSO is given the model setup, which includes data for customer loads, damage functions, commodity prices, carbon costs, DERs, climate, grid reliability, and grid emissions; (ii) the PSO passes sizing decisions for gas generators, solar PV, and energy storage to DER-CAM; (iii) after running, DER-CAM passes the full DER fleet with operation to the MCS; and finally, (iv) upon simulating system operation, the MCS communicates the set of outages in the microgrid with the regression model.

\section{Upper level: PSO}

PSO, a metaheuristic optimization method, links DER-CAM and MCS in the lower level. A heuristic approach like PSO is needed to integrate MCS with DER-CAM because of nonlinearities in the formulation of reliability (e.g., the interruption cost is nonlinear). PSO has the flexibility to embed distinct calculations from mathematical programming and simulation methods because it requires few assumptions about the problem, e.g., about continuity and differentiability. ${ }^{43}$ We use PSO, and not other heuristics, because PSO finds application primarily among nonlinear, continuous-discrete optimization problems, of which the reliability-based microgrid planning problem is one. ${ }^{44}$

We follow the conventional PSO formulation that is standard in the literature ${ }^{45}$-and add to it only diversity-based termination 
criteria, as we will explain. The remainder of this section summarizes the PSO's formulation, parameters, and implementation.

a. Formulation. PSO is population-based, iterative, and stochastic. The population consists of particles $i \in\left\{1, \ldots, n_{\text {particles }}\right\}$, defined by a position $x_{i d}$ and velocity $v_{i d}$ over $n_{\text {dim }}$ dimensions in the solution space, where each dimension $d \in\left\{1, \ldots, n_{\text {dim }}\right\}$ defines a single decision variable. Particles move through the solution space, solve the problem for a location in the space (a candidate solution), and store and share the solution value, or "fitness," $f$. By interacting, particles exploit areas around better solutions, which are those that minimize the objective function-here, the total cost defined in Eq. (14).

Dimensionality in the bilevel model includes numbers of discrete generators, solar PV capacity, and battery storage capacity. Upper and lower bounds for each dimension, $\bar{x}_{d}$ and $\underline{x}_{d}$, constrain the solution space. The maximum velocity is set to allow particles to traverse the entire solution space in a single iteration. When velocities carry particles outside the space, an absorption boundary method returns escaped particles to the position from which they exited.

The PSO iterates by updating particle velocity $v_{i d}$ and position $x_{i d}$ on a dimension-by-dimension basis. Given an initial randomization $v_{i d}^{(1)}$ and $x_{i d}^{(1)}$ for each dimension $d$,

$$
\begin{gathered}
v_{i d}^{(t+1)}=w v_{i d}^{(t)}+c_{1} R_{1}\left(p_{i d}-x_{i d}^{(t)}\right)+c_{2} R_{2}\left(p_{g d}-x_{i d}^{(t)}\right), \\
x_{i d}^{(t+1)}=x_{i d}^{(t)}+v_{i d}^{(t+1)},
\end{gathered}
$$

where $i, d$, and $t$ index the particle, dimension, and iteration, respectively, $p_{i d}$ is the best position found by particle $i$, and $p_{g d}$ is the best position found by the entire population. $R_{1}$ and $R_{2}$ are uniformly distributed random numbers in the interval $[0,1]$; hence, particle motion is in part stochastic. The three weighting coefficients $w, c_{1}$, and $c_{2}$ define the updating process. The inertia weight $w$ balances local and global search, ${ }^{45}$ where higher values (e.g., 0.9) maintain particle motion and facilitate global exploration and lower values (e.g., 0.4) cause exploitation of local optima found so far. ${ }^{46}$ The terms $c_{1}$ and $c_{2}$ act as attraction parameters, pulling particle trajectories toward local and global best positions, respectively.

b. Parameterization. We use a global best particle topology, in which all particles communicate the global best position $p_{g d}$ and set the population size to six particles, inertia $w$ to the standard value of 0.7298 , and attraction parameters $c_{1}$ and $c_{2}$ to the standard value of 2.9922. Finally, we set the initial position for three particles to ensure a diversity of starting conditions, forcing one to near-zero investment, another to a reliable generator-based configuration, and the final one to a reliable solar PV- and battery storage-based configuration. As is typical with heuristics, our parameterizations are based on our experience with the problem.

c. Implementation. The PSO is implemented in three main steps (Fig. 2). The first is initialization or, for later iterations, updating. Second, after correcting positions $x_{i d}$ if needed (via boundary absorption), the PSO evaluates particle fitness $f_{i}^{(t)}$. Each particle maintains a record of its best solution found so far, pbest $t_{j}$, as well as of the global best fitness gbest, both of which guide particle trajectories. The final step is a check on convergence. Traditionally, PSO algorithms are set to terminate upon reaching a maximum number of iterations or when failing to improve gbest for a succession of iterations, both of which are prescribed. We employ setting both the maximum number of iterations to 70, which we find is sufficient for the PSO to reach convergence, and the stall threshold to three. We also use diversity-based termination criteria for swarm velocity and position. ${ }^{47}$ The normalized mean velocity diversity $D_{\text {Velocity }}^{(t)}$ and normalized mean position diversity $D_{\text {position }}^{(t)}$ at iteration $t$ are given by

$$
\begin{gathered}
D_{\text {velocity }}^{(t)}=\frac{1}{n_{\text {particles }}} \frac{1}{n_{\text {dim }}} \sum_{i} \sum_{d} \frac{\left|v_{i d}^{(t)}\right|}{\bar{x}_{d}-\underline{x}_{d}}, \\
D_{\text {position }}^{(t)}=\frac{1}{n_{\text {particles }}} \frac{1}{n_{\text {dim }}} \sum_{i} \sum_{d}\left(\frac{x_{i d}^{(t)}-p_{g d}}{\bar{x}_{d}-\underline{x}_{d}}\right)^{2} .
\end{gathered}
$$

The position diversity is measured relative to the current gbest position $p_{g d}$. As such, these measures indicate whether and when the swarm has converged to a common minimum in the solution space and ceased widespread exploration. Based on the experience with the problem, we further require that $D_{\text {Velocity }}^{(t)}<0.1$ and $D_{\text {position }}^{(t)}<0.05$ for three successive iterations before terminating.

\section{Lower level: DER-CAM}

DER-CAM is LBNL's platform for microgrid investment planning modeling. We provide cost terms that comprise the objective function in Sec. II B. Because the complete formulation is large, we direct the reader to Ref. 26 for full details.

We tune DER-CAM to solve two pieces of the investment planning problem. First, given PSO sizing decisions for gas generators, solar PV, and battery storage, we let DER-CAM size thermal resources (natural gas chillers, absorption chillers, and thermal storage). Second, we have it solve the scheduling problem, in which it determines optimal operating schedules for all units and decides if and when to purchase utility electricity and natural gas. Its solution minimizes the operating cost [Eqs. (8) and (15)].

DER-CAM calculates investment, operating, and interruption costs and also treats reliability via a constraint-what we called the "resource adequacy constraint" in our previous work $^{42}$-which requires that the sum of generator capacity, average solar PV output, and capacity of one discharge cycle from electric storage exceed the critical load. Because we employ MCS and LBNL's customer damage functions, we remove the analogous elements of DER-CAM (the interruption cost, resource adequacy constraint, and VOLL parameter) and thus use DER-CAM primarily for its scheduling algorithm.

\section{Evaluating reliability via sequential MCS}

MCS is widely used for reliability evaluation in engineering and power systems. The sequential MCS routine used in the bilevel model has been developed previously by us; ${ }^{32}$ we direct the reader there for detail but here continue with an overview of its functionality. Broadly, our MCS method tailors general approaches for bulk grid reliability evaluation to the case of grid-connected microgrids by including microgrid-specific resources and a flexibility constraint, which is the requirement that aggregate DERs ramp between time steps to meet fluctuating loads. Resource ramping limits can be critical to the analysis, as we showed in Ref. 32 . 
a. Functionality. MCS evaluates reliability by simulating random failures in grid and DER operation. It repeats year-long simulations, each identical except for a distinct set of random failures and repairs. The goal is to find the timing and duration of outage sequences within the microgrid-so-called "loss of load" events where the load is not met-and three reliability indices, in particular: (i) the loss of load expectation (LOLE) or expected hours of outage per year, (ii) the loss of load frequency (LOLF) or number of loss of load events per year, and (iii) the loss of load cost (LOLC)-i.e., the interruption cost [Eq. (13)]. In the bilevel model, the MCS communicates the set of interruptions with the LBNL regression model to calculate LOLC. Year-long simulations are repeated until reliability indices converge to the expected values, whereupon the MCS returns the expected interruption cost or average over all years, for use in Eq. (14).

b. Reliability indices. LOLE and LOLF are aggregate measures of distinct loss of load events and thus serve as appropriate metrics that summarize the level of reliability provided by a microgrid. ${ }^{40}$ LOLF is analogous to SAIFI, while the loss of load duration (LOLD; given by LOLE/LOLF) is analogous to CAIDI (customer average interruption duration index). SAIFI and CAIDI are standard customer-level reliability indices reported by distribution utilities. The LOLC is the aggregate measure of the economic losses of distinct loss of load events and thus serves as an appropriate cost metric for unreliability against which the benefit of microgrid investment can be assessed.

\section{Valuing reliability via customer damage functions}

The LBNL regression model ${ }^{33,48,49}$ defines the customer damage functions $c_{k}\left(d_{i}\right)$ used in Eq. (13). LBNL's work-the most comprehensive on the topic-standardizes the results of numerous interruption cost surveys spanning several U.S. utilities and decades. (See, e.g., Refs. 50-52, which list studies, as well as Ref. 53 for detailed discussion on an early survey.) In these surveys, customers estimate their own economic losses for various outage scenarios. Though surveys have some downsides, e.g., unknown human elements, survey bias, and inaccurate predictions, they are widely considered an appropriate approach for estimating losses.

The customer damage functions $c_{k}\left(d_{i}\right)$ define economic losses as a function of interruption type and customer type. Customers are defined by, e.g., class and size, and interruptions are defined by duration, time of day, and season. Damage functions therefore capture time-dependent variation in losses that are readily convolved with the MCS's outage sequences and provide more details about losses and a finer level of granularity than that of the VOLL point estimates used in analytical reliability methods. It is these damage functions, given in Ref. 33, which we apply in the bilevel model. For generality, in the case studies that follow (Sec. IV), we apply the more general tabular estimates from the LBNL regression model (reported in the supplementary material), which average costs across customer classes.

\section{Data}

Underlying the model are numerous datasets that define any particular model run-such as for customer loads and damage functions, commodity prices and electric tariff schedules, the cost of carbon, and technology data, climate data, and bulk grid data on marginal emissions and reliability. These are reported in the supplementary material.
In Sec. IV, we model three building types that vary in size and load shape: a hospital (large C\&I customers with a peak electric load of $1414 \mathrm{~kW}$ and an annual consumption of $9.3 \mathrm{GWh}$ ), medium-sized office building (small C\&I, $174 \mathrm{~kW}, 0.6 \mathrm{GWh}$ ), and secondary school (medium C\&I, $927 \mathrm{~kW}, 3.1 \mathrm{GWh}$ ). All loads are taken to be criticali.e., it must be supplied during outages. Load data are from the DOE commercial reference building stock and include electric, cooling, heating, and natural gas loads. ${ }^{54}$ We consider deployment in southern California, using climate data from the Camp Pendleton TMY3 location (site number 722 926), ${ }^{55}$ with interconnection to the north coast district in the San Diego Gas and Electric service territory, a reliable district with SAIFI of 0.461 occurrences per year (occ/yr), MAIFI of $0.239 \mathrm{occ} / \mathrm{yr}$, and SAIDI of $50 \mathrm{~min} / \mathrm{yr}$ (Ref. 56)

\section{VALIDATION AND RESULTS}

\section{A. Validation without reliability}

The goal of the validation is to compare total cost (gbest) values found by the PSO with those calculated by DER-CAM. Classical validation would require data on real-world system adoption, with the known solution and annual costs. Because we know of no such dataset, we compare solutions with those made by DER-CAM, a reputable investment planning model. The two models are inherently different in their full form due to their treatment of reliability, so validation must neglect it. We remove all reliability elements-the resource adequacy constraint from DER-CAM and the interruption cost, MCS, and regression model from the bilevel model. What remains are models with identical objective functions but different engines that provide DER sizing.

We perform validation on the three building types noted in Sec. III C. Each setup with the bilevel model is repeated 100 times to capture random particle search behavior and compared with the single DER-CAM solution, for which we assign a very restrictive optimality gap of $5 \times 10^{-4}$.

Figure 3 presents the results of the validation. Total cost values are consistent, with an average difference between DER-CAM and the PSO of $1.1 \%, 3.5 \%$, and $2.2 \%$ for the hospital, office building, and school, respectively. On occasion, the PSO returns an outlier solution: $6 \%$ of solutions for the office building and $2 \%$ for the school have gbest values that are at least $10 \%$ larger than the DERCAM solution. The worst gbest value for the hospital is $3 \%$ larger. When these few outliers are omitted, the average total cost difference for the office building drops from $3.5 \%$ to $1.0 \%$. As it is, the observed PSO performance is sufficient because we are studying the problem systematically and are interested in "next-best" solutions and local optima, in particular those solutions with only slightly larger gbest (total cost) but perhaps radically different $p_{g d}$ (DER selection), as we will show later in Fig. 7.

The DER-CAM solution and best PSO solution over the 100 runs match very closely, with differences of $0.2 \%, 0.0004 \%$, and $0.005 \%$, respectively, indicating that the PSO is successful in identifying the "ground truth" DER mix found by DER-CAM, a reputable MILP model. We therefore claim that this stripped down version of the bilevel model (i.e., without reliability) is successful as a sizing engine and hence provides a proper basis for adding reliability, to which we now turn. 


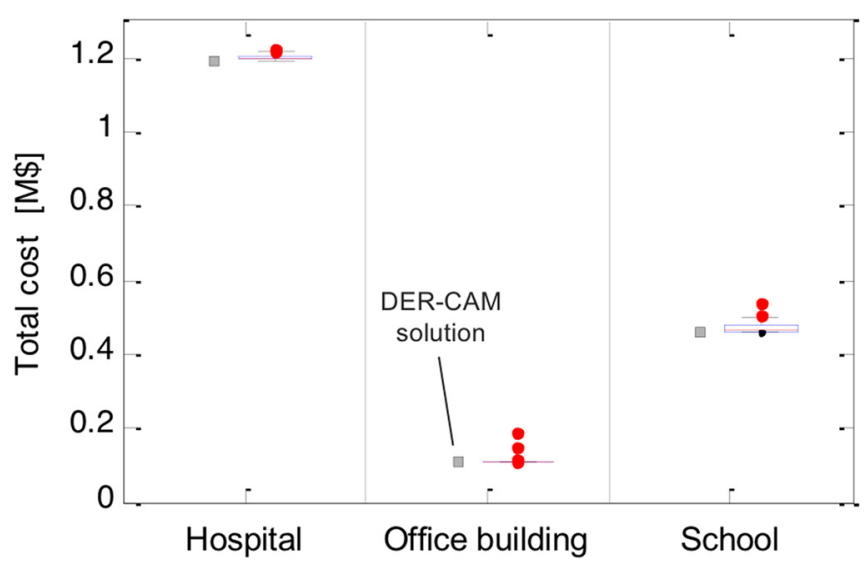

FIG. 3. Validation of the bilevel model against DER-CAM, with reliability elements removed from each model to enable like-to-like comparison. Validation compares the total cost found by the bilevel model (i.e., gbest fitnesses) over 100 repeated runs (box plot in each pair with dots as outliers) with the total cost calculated by DER-CAM (square mark). Fitnesses match closely. The PSO identifies the area of the DER-CAM optimal solution in $100 \%, 94 \%$, and $98 \%$ of runs for the hospital, office building, and school, respectively.

\section{B. The effects of adding reliability}

Here, we explore the different effects of integrating reliability as a cost in the objective function vs as a constraint on investment. For the former, we use the bilevel model with its interruption cost, sequential MCS algorithm, and customer damage function parameter. For the latter, we use DER-CAM with its resource adequacy constraint (discussed in Secs. I and III B 2). We do not make comparisons to DERCAM with its interruption cost, prescribed outages, or VOLL because these model elements were added to DER-CAM after we had acquired the source code and begun our work. (Discussion and qualitative comparison to DER-CAM with its full array of reliability elements is given in Sec. IV D.)

To explore the effects of reliability, we ask: how does differing treatment affect cost-optimal investment and, further, the cost-benefit outlook, which underlies any decision to invest (or not)? We model the hospital building type to demonstrate. Because cost-benefit analysis requires comparing against the baseline of not investing, we model a utility customer and microgrid customer that represent the pre- and post-investment cases, respectively. The two are identical in all respects except investment in DERs. We define the economic benefit from investing to be the difference in the total cost between the two and to be positive when the microgrid reduces the utility customer total cost.

Figure 4 shows costs and investment for six separate cases that define how reliability is treated. Two cases with DER-CAM first omit ("No RA") and then include ("With RA") the resource adequacy (RA) constraint, while four cases with the bilevel model vary the customer damage function $c_{k}\left(d_{i}\right)$ underlying the interruption cost. Given the damage function $b \cdot c_{k}\left(d_{i}\right)$, we model the set $b=\{0,1,10,20\}$, which represents, e.g., hospitals with null, nominal, intermediate, and high valuations of the need for reliable electric service. While it is not possible to attribute these cases to any single hospital (because the LBNL regression functions are segment averages), the range $b=\{0,1,10,20\}$ reflects the different types of hospitals for which reliability is varyingly critical-e.g., rural hospitals providing only standard services, hospitals providing specialized services and critical care, and hospitals acting as regional trauma centers. The range in $b$ is plausible because the LBNL regression functions report variation in $c_{k}\left(d_{i}\right)$ of several orders of magnitude. The value $b=0$ (no need for reliability) probably does not map to any hospital, but we include it as a logical extreme for the purpose of comparing the two models.

For the cases without reliability, the two models match closely, as expected from the validation. In the No RA case, DER-CAM calculates a total microgrid cost of $\$ 1.19 \mathrm{~m}$ and an economic benefit of $24 \%$, while the bilevel model finds them to be $\$ 1.20 \mathrm{~m}$ and $23 \%$ when $b=0$.

Clear differences emerge, however, upon adding reliability. In DER-CAM, the primary effect of adding the resource adequacy constraint (With RA case) is on battery investment, which increases from $0 \mathrm{MW}$ to $1.2 \mathrm{MW}$. Investment in generators and PV is unchanged. The associated economic impact is material: the additional battery capacity increases the investment cost $49 \%$ (from $\$ 0.28 \mathrm{~m}$ to $\$ 0.41 \mathrm{~m}$ ) and decreases the economic benefit 29\% (from $\$ 0.38 \mathrm{~m}$ to $\$ 0.27 \mathrm{~m}$ ).

The analogous change in the bilevel model-increasing customer damage costs from zero to their nominal value of $b=1$ - has almost no effect on median investment: solar PV capacity decreases by $11 \mathrm{~kW}$ (1.9\%), while generator and battery capacities remain unchanged. Changes in the cost are also modest: the investment cost increases $2.6 \%$, the total cost increases $2.2 \%$, and the economic benefit decreases $0.1 \%$. These changes are not driven by a prescribed minimum threshold for DER investment, as with the resource adequacy constraint, but rather reflect this customer's particular valuation of reliability defined in $b \cdot c_{k}\left(d_{i}\right)$. Here, that valuation is not so large as to drive material increase in investment nor significant changes to the cost-benefit analysis.

Customer damage functions increased 10 -fold $(b=10)$, by contrast, increase the interruption cost significantly. In response, the bilevel model selects slightly more generator and solar PV capacity in the cost-optimal configuration. Note, however, that the additional capacity does not necessarily drive the interruption cost to zero because the bilevel model finds a cost-optimal trade-off between the investment cost and interruption cost. The valuation $b=20$ illustrates a case in which a high valuation of reliability leads to significant increases in cost-optimal investment-increases that subsequently reduce the interruption cost to near-zero but at the expense of a much higher investment cost.

Table II summarizes the results and compares differences in costs, benefit, and investment across the two models. Differences are significant. Though $b=\{1,20\}$ might represent two different classes of hospitals with distinct estimates of reliability worth, DER-CAM's constraint-based approach can provide at most a single estimate for either-via the With RA case. Yet, DER-CAM over-estimates costoptimal investment by up to $22 \%$ relative to the $b=1$ case (in fact by infinity for battery storage) and under-estimates it by up to $56 \%$ relative to the $b=20$ case. Consequently, DER-CAM over-estimates the investment cost by $50 \%$ when $b=1$ and under-estimates it by $32 \%$ when $b=20$. Ultimately, it under-estimates the economic benefit by $27 \%$ and $47 \%$ for the two cases, respectively.

As the six cases demonstrate, the means by which reliability is added-either as a constraint or objective — can radically affect investment decision-making and the cost-benefit outlook. With a constraint-based approach, the option to include reliability is binary: the resource adequacy constraint is either included or not, with "optimal" configurations therefore taking one of the two forms. By its 

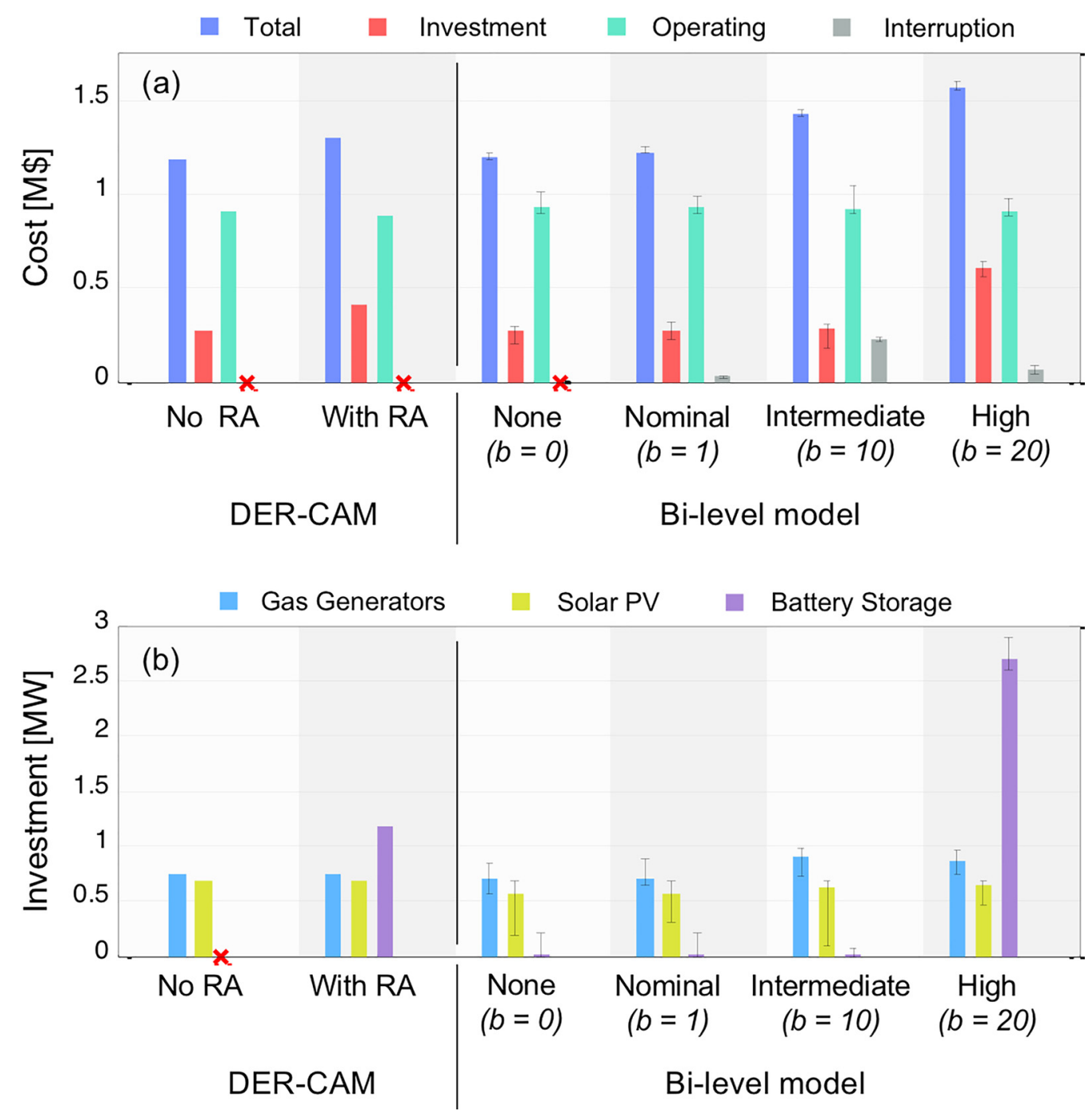

FIG. 4. The (a) costs and (b) configuration for six reliability cases. Two cases modeled in DER-CAM first omit ("No RA") and then include ("With RA") DER-CAM's" resource adequacy constraint. Four cases using the bilevel model vary the customer damage function $b \cdot c_{k}\left(d_{i}\right)$, setting $b=\{0,1,10,20\}$. The 100 runs from the validation are used for $b=0$, while new cases for $b=\{1,10,20\}$ are run 25 times to capture variation in random PSO search. Thick bars give the median, and uncertainty bars give the 5 th and 95 th percentiles. "X" marks denote the values of zero. A constraint-based treatment of reliability limits configurations to one of two outcomes: "No RA" or "With RA". The bilevel model, by contrast, allows for continuous variation in investment and costs as driven by the customer's valuation of reliability inherent in $b \cdot c_{k}\left(d_{i}\right)$.

nature, DER-CAM's resource adequacy constraint at most increases investment and reduces the economic benefit of investing. This is because a more reliable microgrid does not reduce any associated cost in the objective function. A clear advantage of a cost-based approach to reliability is that additional investment can reduce the total system cost by mitigating the interruption cost, and further that such effects are continuous rather than binary: optimal DER capacities and costs will increase gradually as the value placed on reliability, i.e., $b$, increases.

Figure 5 provides a cost-disaggregated look at the cost-benefit analysis for the hospital building type for the With RA and $b=20$ cases. It demonstrates how the cost-benefit analyses are fundamentally different in the two models, which helps explain why large differences in investment emerge between the two models. In both models, the utility customer has an identical operating cost. The key difference between the two is the interruption cost, which in this case is material (about one-third of the utility customer operating cost). With the interruption cost, the utility customer total cost in the bilevel model is larger than the equivalent in DER-CAM, and so the starting point for cost-benefit analysis is shifted; that is, there is greater imperative in the bilevel model to invest in DERs to reduce utility customer costs. In this example, the bilevel model returns a cost-optimal microgrid with a $47 \%$ larger investment cost, nearly equal operating cost, and $21 \%$ larger total cost than the DER-CAM equivalent. Yet, the bilevel model reports an economic benefit that is $87 \%$ greater.

Such differences are potentially profound. Though in this example, both models find investment to be economic, in other cases 
TABLE II. Cost, benefit, and investment for select reliability cases for the hospital building type. Values reported for bilevel cases are the median of 25 runs. Values in parentheses give the percent difference between the DER-CAM With RA case and the particular bilevel model case.

\begin{tabular}{lccc}
\hline \hline & DER-CAM & \multicolumn{2}{c}{ Bilevel model } \\
\cline { 3 - 4 } & with RA & $b=1$ & $b=20$ \\
\hline Cost and benefit (million dollars) & & \\
$\quad$ Total cost & 1.30 & $1.23(6.1)$ & $1.58(-18)$ \\
Investment cost & 0.41 & $0.24(50)$ & $0.60(-32)$ \\
Economic benefit & 0.27 & $0.37(-27)$ & $0.50(-47)$ \\
Investment (megawatts) & & & \\
Gas generators & 0.75 & $0.71(5.6)$ & $0.86(-13)$ \\
Solar PV & 0.69 & $0.56(22)$ & $0.65(5.8)$ \\
Battery storage & 1.18 & $0($ Inf $)$ & $2.70(-56)$ \\
\hline \hline
\end{tabular}

investment may appear uneconomic unless interruption costs are explicitly considered. Adding the cost and benefit of reliability adds information to the problem at hand and serves only to strengthen the case for investment because interruption costs cannot increase upon investing. Models that treat reliability explicitly as a cost-based objective can therefore demonstrate positive economic benefit where models limited to reliability constraints may not.

\section{Comparisons of the PSO solution set and the DER- CAM solution}

The PSO returns numerous solutions in its search for gbest and $p_{g d}$ producing as a result a varying but valuable solution set. Some are

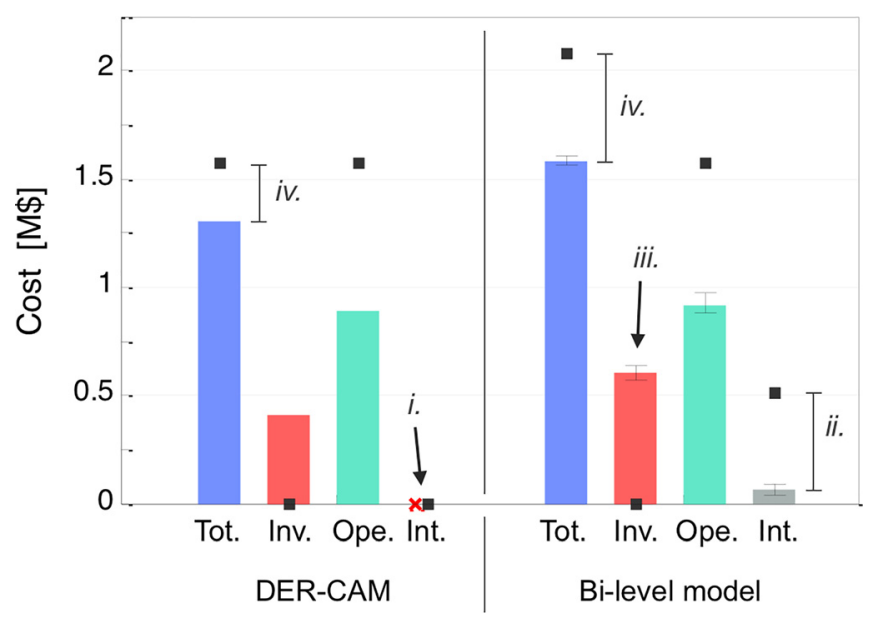

FIG. 5. Cost breakdown and economic benefit for the DER-CAM With RA case and bilevel model $b=20$ case. Thick bars denote the median costs for the total "tot," investment "inv," operating "ope," and interruption "int" cost, and uncertainty bars give the 5th and 95th percentiles. Utility customer costs are shown with squares. Comparing across models shows how the cost-benefit analysis is fundamentally different in the two models. Because it does not have an interruption cost (i), DER-CAM reports a lower total cost for both customer types compared to the bilevel model. By contrast, the bilevel model seeks to reduce an interruption cost (ii) by investing in greater DER capacity relative to DER-CAM (iii). As a result, the bilevel model reports not only a $47 \%$ larger total cost but also an $87 \%$ larger economic benefit from investing (iv). poor, but many have only incremental difference in sizing and similar total cost (e.g., as observed in Fig. 4 for $b=\{0,1,10,20\}$, which shows wide variation in sizing but small variation in the total cost for gbest solutions). Figure 6 goes a level deeper, showing an additional set of information: individual costs for the full solution set (i.e., $f_{i} \forall_{i}$ in addition to gbest) for one run with $b=20$ in the previous analysis of the hospital building type. Sorting the solution set by the investment cost shows how configurations with marginally larger DER capacities can reduce interruption costs. It also shows how investment can be misdirected, as when it does not reduce interruption costs (as observed for the two anomalous results-candidate solutions \#83 and \#100-which lack battery storage and have a high total cost, an operating cost, and an interruption cost relative to surrounding solutions). The full solution set helps to explain the variation of solutions shown in Fig. 7.

Perhaps most importantly, the PSO solution set can be used to assess the relative quality of the optimal solution reported by DERCAM and the bilevel model. Figure 7 shows the solution set for the hospital building for $b=20$ as it lies in the reliability-cost space. The level of reliability is reported as the LOLE metric, the expected number of hours of outage per year. With its bad and good solutions, the bilevel model traces the start of a "feasibility region," i.e., the set of possible outcomes for cost and reliability pairs. It also provides an "optimality front" of best outcomes, i.e., a set of nondominated solutions for which an improvement in reliability incurs the smallest increase in the total cost.

Two reliability-cost spaces are shown. The first, in Fig. 7(a), shows the cost-reliability trade-off for the total cost less interruption cost, which is the total cost defined by DER-CAM. We remove the interruption cost from bilevel model solutions to enable like-to-like comparisons, without an interruption cost nor knowledge of the value

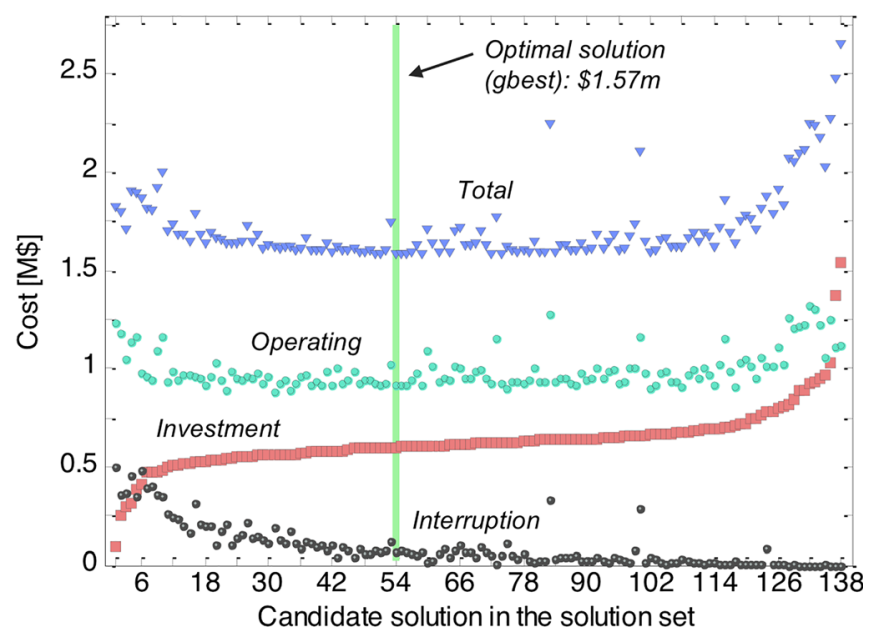

FIG. 6. Individual costs sorted by the investment cost for every location $x_{i d}^{(t)}$ in the solution space solved during the PSO's search for optimality. A single case for the hospital building type, with six particles solved over 23 iterations (138 candidate solutions), is shown. The optimal solution, $p_{\text {gd, }}$, has a gbest value of $\$ 1.57 \mathrm{~m}$. On either side are suboptimal regions: on the left, under-investment with high interruption costs; on the right, over-investment with costly redundant capacity. The solution set is reminiscent of the classic depiction of power system planning, where outage costs decrease with additional investment, thereby producing a quadratic total cost curve. 
of reliability $c_{k}\left(d_{i}\right)$, the nondominated set acts as a Pareto front, which hosts the subset of solutions that are optimal in the multiobjective sense; i.e., they give the optimal trade-off between cost and reliability, two competing objectives. Without the knowledge of $c_{k}\left(d_{i}\right)$, a system developer would in theory choose a solution on the nondominated set with the preferred combination of cost and reliability. The gbest solution lies on the front, but the DER-CAM solution lies off of it and is therefore suboptimal. Other solutions that roughly match the DERCAM solution have better reliability at the same cost (graphically, the nondominated set directly below the DER-CAM solution), an equivalent level of reliability at a lower cost (directly to the left), or both.

The second space, in Fig. 7(b), shows reliability vs total cost as defined in the bilevel model. The y-axis in this second space is identical to that in Fig. 7(a), while the $\mathrm{X}$-axis is translated from Fig. 7(a) by adding the interruption cost. We calculate LOLE and the interruption cost for DER-CAM's optimal configuration post-hoc using the MCS and LBNL regression model. The gbest solution, which has a total cost of $\$ 1.57 \mathrm{~m}$ and a LOLE value of $0.18 \mathrm{~h} \mathrm{yr}^{-1}$, marks the beginning of the nondominated set. Above it, configurations are less reliable and costlier. Below it, reliability can be increased but at increasing cost, though these solutions are known to be suboptimal given knowledge of $c_{k}\left(d_{i}\right)$. The configuration that DER-CAM finds to be optimal is less reliable (LOLE of $1.10 \mathrm{~h} \mathrm{yr}^{-1} ; 600 \%$ greater) and more expensive ( $\$ 1.73 \mathrm{~m} ; 10 \%$ greater) than that returned by the bilevel model.

That the DER-CAM solution is inferior generally to numerous bilevel model solutions (even suboptimal ones) is unsurprising. The bilevel model has been built specifically to address reliability in the investment planning problem. Relative to DER-CAM's constraintbased approach, it has additional information about system costs and optimizes configurations to reduce them. In the worst case, the bilevel model will provide an equally informed solution. When interruption costs are material, which is likely given the nature of microgrids, the bilevel model can return a radically different optimal solution from that provided by DER-CAM-one that is better informed and therefore a more accurate estimate of the true costs and benefits of deployment.

\section{Comparisons against DOE models with a reliability cost}

In addition to quantitative comparisons made against DERCAM's constraint-based approach, of interest are comparisons against the DOE models that formulate reliability as a cost-i.e., DER-CAM, REopt, and MDT. Due to the challenges associated with obtaining source codes, configuring scenarios, and running cases-a task beyond the scope of this paper, which reports on building a new model-we are unable to offer a quantitative comparison here. Though we leave that for future work, here we discuss two areas in investment planning modeling where model intercomparison would be particularly useful. One is the estimates that define customer losses from outages, i.e., VOLL and customer damage functions. The second is the set of model parameters, such as bulk grid and DER failures, which comprise the reliability calculation. Differences in these affect the perceived costs of outages and underlying reliability, respectively.

A standard approach of MILP models-and taken by DER-CAM and REopt-is the use of a grid outage parameter, in which the modeler prescribes grid outages of desired timing and duration, in concert with a VOLL parameter, in $\$ / \mathrm{kWh}$, assigned to the load that goes unserved during grid outages (due to insufficient DER capacity). The objective function includes an interruption cost that is the product of the VOLL and unserved load, in kilowatt-hours, and the model therefore decides whether it is more economic to forego DER investment and incur interruption costs or to invest and incur investment costs. This approach is useful because it frames the cost-reliability trade-off endogenously. However, it makes several simplifications in order to fit with the MILP framework: it uses a VOLL (a scalar value) for its damage cost instead of the more granular customer damage functions, which report economic losses as a function of outage timing and duration. In reality, customer damages vary by hour of the day, day of the
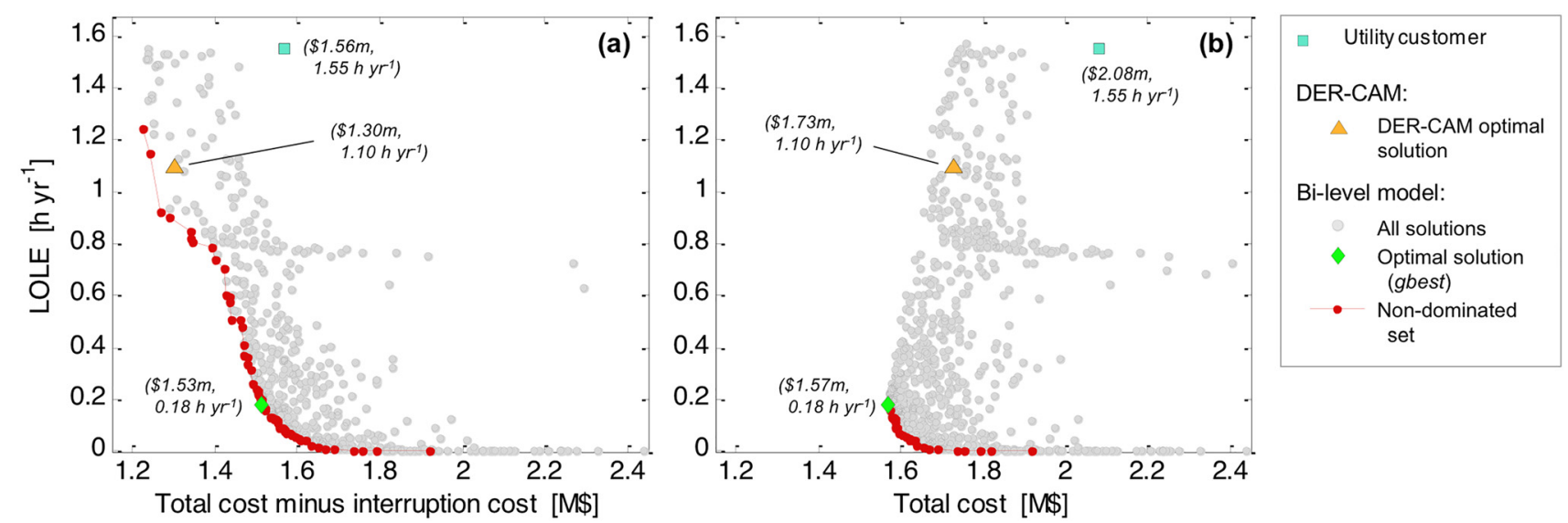

FIG. 7. The loss of load expectation (LOLE; the expected hours of outage per year) vs (a) investment plus operating costs (i.e., total cost less interruption cost) and (b) total cost. Shown is the hospital building type with $b=20$, with all individual solutions returned by the PSO in its search for optimality, the gbest solution, the DER-CAM optimal solution, and the utility customer solution. The non-dominated set is the subset of solutions that provide a best-case combination of cost and reliability and for which incrementally better reliability incurs the least additional cost. The PSO-in returning numerous solutions, both good and bad-traces the start of a feasibility region in the reliability-cost space that allows one to compare the relative quality of the DER-CAM and bilevel model solutions. Because the DER-CAM solution lies off the nondominated set in (a) and because it incurs a higher total cost and is less reliable as seen in (b), it is inferior to the optimal solution found by the bilevel model. 
week, and season-variation that is captured by damage functions but not a VOLL. Another simplification concerns outage prescription. REopt treats only a single outage of average duration, e.g., using CAIDI, but simulates its occurrence at every hour of the year to calculate the duration that the microgrid can maintain power supply during the outage-a calculation aligned more with resiliency than reliability. In reality, outages vary considerably in duration and number across years. DER-CAM can consider multiple outages, but the task of prescribing them is onerous given the huge number of permutations in outage frequency and duration. Modelers may need to guess and prescribe worst-case scenarios, e.g., outages that occur during times of peak load. The challenge of handling a large number of permutations is further compounded by the need to consider random DER failures and scheduled maintenance in concert with grid outages.

The MCS approach used by the bilevel model is a panacea for these concerns. Its core function is to simulate thousands of permutations of outages, given the knowledge of the failure and repair rates of the bulk grid and DERs. It convolves these point failures with timevarying customer damage functions and time-varying loads until statistical convergence among permutations is reached. While a high degree of user subjectivity is required to parameterize outages in DERCAM and REopt, the MCS in the bilevel model requires only parameterization of underlying failure and repair rates, while also allowing for prescribed outages and maintenance schedules. We therefore expect the bilevel model, with its use of MCS, to produce a more statistically robust set of outages (timing and duration), better estimates of the costs of those outages, and therefore a more representative estimate of the interruption cost that drives investment decision-making.

The MDT model, like the bilevel model, is a hybrid of MILP, heuristic optimization, and simulation. It combines two separate models: the first is a tuned version of DER-CAM (without reliability) and determines a baseline DER mix, while the second integrates a genetic algorithm and simulation-based reliability evaluation to refine the baseline DER mix. Refinements include the selection of specific manufacturer units, placement of DERs within a network, and deployment of power system equipment to integrate DERs. With respect to its calculation of reliability, MDT is functionally similar to the bilevel model because it simulates random grid and DER outages and calculates LOLF and LOLE. The key difference lies with where reliability is integrated. In MDT, reliability is considered in the second model after core DER selections are first made without reliability. As such, reliability is exogenous to DER selection and therefore drives results for power system network design and DER placement but not for DER selection. We therefore expect that MDT's selection of DERs would closely resemble the DER-CAM solution in Fig. 7. A more robust and accurate approach, as taken by the bilevel model, would be to integrate the reliability cost with all other system costs in the objective function of a single optimization framework, thereby making reliability endogenous to DER selection. If the MDT model were to incorporate the newer version of DER-CAM that includes a reliability cost, it would resemble DER-CAM (coupled with a second model for refinement) and be subject to the same challenges noted previously.

Whether and to what extent the bilevel model and three DOE models find differences in investment, reliability, cost, and benefit is, however, unknown. A systematic effort to explore the implications of differences in the models could shed meaningful light, perhaps uncovering hidden nuances in how various reliability elements drive model results. To this end, we present in the supplementary material the datasets underlying the case studies presented in this section. Of importance in a model intercomparison study is the exploration of the sensitivities of reliability parameters, as sensitivities (and risk) are a central issue for microgrid investment in real-world commercial settings.

\section{CONCLUSION}

This work presented a new microgrid investment planning model, an engineering-economic model that determines optimal sizing and scheduling for DERs in microgrids. It is built to use best-available methods and data for evaluating reliability, namely sequential MCS and customer damage functions. MCS simulates distinct power outage sequences and a microgrid's ability to island and power itself autonomously.

Through validation, we show that our new model matches DER-CAM for solution quality without reliability elements. We then show in case studies that, with reliability elements added, our model provides a more diverse solution set and a better optimal solution than models which treat reliability through investment constraints. Specifically, we observe improvements in the estimate of reliability by $600 \%$, total cost by $6 \%-18 \%$, investment cost by $32 \%-50 \%$, and the economic benefit of investing by $27 \%-47 \%$. Improvements stem from our model's explicit treatment of reliability as a cost-based objective.

For those developing microgrids in the real world, the new model improves our ability to estimate the real costs and benefits of investing because, compared to existing models, it considers a wider array of real-world phenomena that affect reliability. It therefore offers a more comprehensive analysis with more robust investment decisionmaking.

Our new model opens several avenues for future work. One avenue concerns the risk of grid outages and the ways in which investors and system designers actually build microgrids to mitigate risk. At present, the model uses the expected, or average, set of outages and interruption cost calculated by the MCS. By contrast, a systematic study that uses interruption costs nearer the distribution tails would help uncover the nature of the reliability premium-i.e., the incremental cost that must be paid to withstand even the most severe outages. A second natural extension is to the catalog of outages considered in the model. We have robust data on routine outages that last $0-8 \mathrm{~h}$-as given by the utility reliability metrics SAIFI, MAIFI, and SAIDI—but lack enumerated data or probability distributions for more severe outages that last days, and that perhaps affect the operation of the natural gas network simultaneously. Including long-term outages could reveal vulnerabilities in otherwise well-planned microgrids. Such an extension is particularly relevant given the important role that natural gas may play in future microgrid infrastructure.

\section{SUPPLEMENTARY MATERIAL}

See the supplementary material for the results related to validation, namely the performance of the PSO in its search for the optimal solution. Also included are the many datasets that define the model runs in this paper, such as for customer load and damage functions, commodity prices and electric tariff schedules, the cost of carbon, technology data, climate data, and bulk grid data on marginal emissions and reliability. 


\section{ACKNOWLEDGMENTS}

This work was supported by the Electric Power Research Institute. Part of this research was developed in the Young Scientists Summer Program at the International Institute for Applied Systems Analysis, Laxenburg (Austria), with financial support from the United States National Member Organization.

The authors thank Daniel Huppmann, of the International Institute for Applied Systems Analysis, for insights on the construction of the bilevel model, as well as Ahmed Abdulla, of the University of California San Diego, and Oytun Babacan, of Imperial College London, for helpful comments during review.

\section{REFERENCES}

'A. K. Basu, S. Chowdhury, S. Chowdhury, and S. Paul, "Microgrids: Energy management by strategic deployment of DERs-A comprehensive survey," Renewable Sustainable Energy Rev. 15, 4348-4356 (2011).

${ }^{2}$ M. Soshinskaya, W. H. Crijns-Graus, J. M. Guerrero, and J. C. Vasquez, "Microgrids: Experiences, barriers and success factors," Renewable Sustainable Energy Rev. 40, 659-672 (2014).

${ }^{3}$ S. Parhizi, H. Lotfi, A. Khodaei, and S. Bahramirad, "State of the art in research on microgrids: A review," IEEE Access 3, 890-925 (2015).

${ }^{4}$ M. Stadler, G. Cardoso, S. Mashayekh, T. Forget, N. DeForest, A. Agarwal, and A. Schonbein, "Value streams in microgrids: A literature review," Appl. Energy 162, 980-989 (2016).

${ }^{5}$ State of Connecticut, Department of Energy \& Environmental Protection, https://www.ct.gov/DEep/cwp/view.asp?a=4405\&Q=508780 for "Microgrid Program," accessed February 2019.

${ }^{6}$ State of Massachusetts, Department of Energy Resources, https://www.mass. gov/community-clean-energy-resiliency-initiative for "Community Clean Energy Resiliency Initiative," accessed February 2019.

${ }^{7}$ Massachusetts Clean Energy Center, https://www.masscec.com/communitymicrogrids-program for "Community Microgrids Program," accessed February 2019.

${ }^{8}$ New Jersey Board of Public Utilities, https://www.nj.gov/bpu/pdf/commercial/ TC\%20DER\%20Microgrid\%20Feasibility\%20Study\%20Application\%20 Final.pdf for "Town Center Distributed Energy Resource Microgrid Feasibility Study Incentive Program," accessed February 2019.

${ }^{9}$ California Energy Commission, https://ww2.energy.ca.gov/contracts/PON-14301 for "PON-14-301-Demonstrating Secure, Reliable Microgrids and GridLinked Electric Vehicles to Build Resilient, Low-Carbon Facilities and Communities," accessed February 2019 (2014).

${ }^{10}$ California Energy Commission, https://ww2.energy.ca.gov/contracts/GFO-17302 for "GFO-17-302-Demonstrate Business Case for Advanced Microgrids in Support of California's Energy and GHG Policies," accessed February 2019 (2017).

"New York State Energy Research and Development Authority, https://www. nyserda.ny.gov/All-Programs/Programs/NY-Prize for "NY Prize," accessed February 2019.

${ }^{12}$ Maryland Energy Administration, https://energy.maryland.gov/Pages/ Resiliency-Hub.aspx for "Resiliency Hub," accessed February 2019.

${ }^{13}$ M. T. Burr, M. J. Zimmer, B. Meloy, J. Bertrand, W. Levesque, G. Warner, and J. D. McDonald, "Minnesota microgrids: Barriers, opportunities, and pathways toward energy assurance," Final Report, Minnesota Department of Commerce (2013).

${ }^{14}$ C. Lotspeich, "Resilient microgrids for Rhode Island critical services," Final Report, Rhode Island Office of Energy Resources (2016).

${ }^{15} \mathrm{P}$. Asmus, http://microgridmedia.com/microgrids-without-subsidy-check-cimarket for "Microgrids without Subsidy? Check Out the C\&I Market," accessed May 19, 2017 (2017).

${ }^{16}$ P. Asmus, Market Data: Microgrids (Navigant Consulting, Inc., 2016).

${ }^{17}$ A. Wilson, Microgrid Deployment Tracker 2Q17 (Navigant Consulting, Inc., 2017).

${ }^{18}$ R. Hanna, V. Disfani, J. Kleissl, and D. Victor, "A new simulation model to develop and assess business cases for commercial microgrids," in 2017 IEEE North American Power Symposium (NAPS) (2017).
${ }^{19} \mathrm{R}$. Hanna, "Business cases for microgrids: Modeling interactions of technology choice, reliability, cost, and benefit," PhD dissertation (University of California, San Diego, Department of Mechanical and Aerospace Engineering, 2017).

${ }^{20}$ S. Lahiri, O. Bystrom, R. Fioravanti, and N. Tong, "Microgrid assessment and recommendations to guide future investments," Report No. CEC-500-2015071 (2015)

${ }^{21}$ Green Business Certification Inc., PEER Rating System Version 2.0 (Green Business Certification Inc., 2018).

${ }^{22} \mathrm{~A}$. Chittum and G. Relf, "Valuing distributed energy resources: Combined heat and power and the modern grid," Electr. J. 32, 52-57 (2019).

${ }^{23} \mathrm{C}$. Gamarra and J. M. Guerrero, "Computational optimization techniques applied to microgrids planning: A review," Renewable Sustainable Energy Rev. 48, 413-424 (2015).

${ }^{24}$ R. Billinton and R. N. Allan, Reliability Evaluation of Power Systems (Springer US, 1996).

${ }^{25}$ T. Lambert, P. Gilman, and P. Lilienthal, "Micropower system modeling with homer," in Integration of Alternative Sources of Energy (John Wiley \& Sons, Inc., 2006), pp. 379-418.

${ }^{26}$ A. S. Siddiqui, C. Marnay, J. L. Edwards, R. Firestone, S. Ghosh, and M. Stadler, "Effects of carbon tax on microgrid combined heat and power adoption," J. Energy Eng. 131, 2-25 (2005).

${ }^{27} \mathrm{~J}$. Eddy, N. E. Miner, and J. Stamp, “Sandia's microgrid design toolkit," Electr. J. 30, 62-67 (2017).

${ }^{28}$ N. D. Laws, K. Anderson, N. A. DiOrio, X. Li, and J. McLaren, "Impacts of valuing resilience on cost-optimal PV and storage systems for commercial buildings," Renewable Energy 127, 896-909 (2018).

${ }^{29}$ A. Jimenez, S. G. Haase, and S. Mathur, "Microgrid analysis tools summary," Report No. NREL/PR-5000-70578 (2018).

${ }^{30} \mathrm{G}$. Vallery and M. Stadler, "Fort hunter liggett microgrid conversion," in paper presented at Military \& Government Microgrids Summit, Arlington, Virginia (2015).

${ }^{31} \mathrm{R}$. Billinton and W. Li, Reliability Assessment of Electric Power Systems Using Monte Carlo Methods (Springer US, 1994).

${ }^{32}$ R. Hanna, V. Disfani, and J. Kleissl, "Reliability evaluation for microgrids using crossentropy Monte Carlo simulation," in 2018 IEEE Conference on Probabilistic Methods Applied to Power Systems (2018).

${ }^{33}$ M. J. Sullivan, J. Schellenberg, and M. Blundell, "Updated value of service reliability estimates for electric utility customers in the United States," Report No. LBNL-6941E (2015).

${ }^{34} \mathrm{M}$. Meiqin, J. Meihong, D. Wei, and, and L. Chang, "Multi-objective economic dispatch model for a microgrid considering reliability," in 2010 2nd IEEE International Symposium on Power Electronics for Distributed Generation (2010).

${ }^{35}$ M. E. Khodayar, M. Barati, and M. Shahidehpour, "Integration of high reliability distribution system in microgrid operation," IEEE Trans. Smart Grid 3, 1997-2006 (2012).

${ }^{36}$ M. H. Moradi, M. Eskandari, and S. M. Hosseinian, "Operational strategy optimization in an optimal sized smart microgrid," IEEE Trans. Smart Grid $\mathbf{6}$, 1087-1095 (2015).

${ }^{37}$ A. Khodaei, S. Bahramirad, and M. Shahidehpour, "Microgrid planning under uncertainty," IEEE Trans. Power Syst. 30, 2417-2425 (2015).

${ }^{38} \mathrm{M}$. Quashie, F. Bouffard, and G. Joos, "Business cases for isolated and grid connected microgrids: Methodology and applications," Appl. Energy 205, 105-115 (2017).

${ }^{39} \mathrm{~N}$. Ghorbani, A. Kasaeian, A. Toopshekan, L. Bahrami, and A. Maghami, "Optimizing a hybrid wind-PV-battery system using GA-PSO and MOPSO for reducing cost and increasing reliability," Energy 154, 581-591 (2018).

${ }^{40} \mathrm{~S}$. Wang, Z. Li, L. Wu, M. Shahidehpour, and Z. Li, "New metrics for assessing the reliability and economics of microgrids in distribution system," IEEE Trans. Power Syst. 28, 2852-2861 (2013).

${ }^{41} \mathrm{R}$. Billinton and R. Allan, "Power-system reliability in perspective," Electron. Power 30, 231 (1984).

${ }^{42}$ R. Hanna, M. Ghonima, J. Kleissl, G. Tynan, and D. Victor, "Evaluating business models for microgrids: Interactions of technology and policy," Energy Policy 103, 47-61 (2017).

${ }^{43}$ R. Eberhart and Y. Shi, "Particle swarm optimization: Developments, applications and resources," in Proceedings of the 2001 Congress on Evolutionary Computation (2001), Vol. 1, pp. 81-86. 
${ }^{44} \mathrm{M}$. Clerc, Particle Swarm Optimization (Wiley, 2006).

${ }^{45}$ Y. Shi and R. Eberhart, "A modified particle swarm optimizer," in 1998 IEEE International Conference on Evolutionary Computation Proceedings. IEEE World Congress on Computational Intelligence (1998).

${ }^{46}$ R. Poli, J. Kennedy, and T. Blackwell, "Particle swarm optimization," Swarm Intell. 1, 33-57 (2007).

${ }^{47}$ Y. Shi and R. C. Eberhart, "Population diversity of particle swarms," in 2008 IEEE Congress on Evolutionary Computation (IEEE World Congress on Computational Intelligence) (IEEE, 2008).

${ }^{48}$ M. J. Sullivan, M. Mercurio, and J. Schellenberg, "Estimated value of service reliability for electric utility customers in the United States," Report No. LBNL2132E (2009).

${ }^{49}$ M. J. Sullivan, M. G. Mercurio, J. A. Schellenberg, and J. H. Eto, "How to estimate the value of service reliability improvements," in IEEE PES General Meeting (2010).

${ }^{50} \mathrm{G}$. Tollefson, R. Billinton, and G. Wacker, "Comprehensive bibliography on reliability worth and electrical service consumer interruption costs: 1980-90," IEEE Trans. Power Syst. 6, 1508-1514 (1991).
${ }^{51} \mathrm{C}$.-K. Woo and R. L. Pupp, "Costs of service disruptions to electricity consumers,” Energy 17, 109-126 (1992).

${ }^{52}$ S. Kufeoglu and M. Lehtonen, "A review on the theory of electric power reliability worth and customer interruption costs assessment techniques," in 2016 13th International Conference on the European Energy Market (EEM) (2016).

${ }^{53} \mathrm{G}$. Tollefson, R. Billinton, G. Wacker, E. Chan, and J. Aweya, "A Canadian customer survey to assess power system reliability worth," IEEE Trans. Power Syst. 9, 443-450 (1994).

${ }^{54}$ Department of Energy Commercial Reference Buildings, https://www.energy. gov/eere/buildings/commercial-reference-buildings; accessed September 19, 2017 (2015).

${ }^{55}$ National Solar Radiation Data Base 1991-2005 Update: Typical Meteorological Year 3, https://rredc.nrel.gov/solar/old_data/nsrdb/1991-2005/tmy3; accessed September 19, 2017.

${ }^{56} \mathrm{M}$. Kurtovich and M. Zafar, California Electric Reliability Investor-Owned Utilities Performance Review 2006-2015 (California Public Utilities Commission, 2016). 